\title{
Comparative adsorption of saturated and unsaturated fatty acids at the iron oxide/oil interface
}

\author{
Mary H. Woodt, M. T. Casfordt, R. Steitz ${ }^{\Uparrow}$ A. Zarbakhsh ${ }^{\S}$, R. J. L. Welbournt, Stuart M. Clarket ${ }^{*}$ \\ + Department of Chemistry and BP Institute, Cambridge University, Cambridge, CB2 1EW, UK \\ §School of Biological and Chemical Science, Queen Mary, University of London, Joseph Priestly \\ Building, Mile End Road, London, UK \\ " Helmholtz-Zentrum Berlin für Materialien und Energie, Hahn-Meitner-Platz 1, 14109 Berlin, \\ Germany \\ *corresponding author: stuart@bpi.cam.ac.uk
}

\begin{abstract}
A detailed comparison of the adsorption behaviour of long straight chain saturated and unsaturated fatty acids at the iron oxide/oil interface has been considered using a combination of surface study techniques. Both depletion isotherms and polarised neutron reflectometry (PNR) show that the extent of adsorption decreases as the number of double bonds in the alkyl chains increases. Sum frequency generation spectroscopic measurements demonstrate that there is also an increase in chain disorder within the adsorbed layer as the unsaturation increases. However, for the unsaturated analogues, a decrease in peak intensity is seen for the double bond peak upon heating, which is thought to arise from isomerisation in the surface-bound layer. The PNR study of oleic acid adsorption indicates chemisorbed monolayer adsorption, with a further diffuse reversible adsorbed layer formed at higher concentrations.
\end{abstract}




\section{Introduction}

The adsorption mechanisms of small organic molecules such as fatty acids on industrially-pertinent metal surfaces has been of considerable interest in recent years, due to their universallyacknowledged efficacy as engine oil additives, particularly as lubricants known as 'friction modifiers' that protect surfaces in the boundary lubrication regime; these are supposed to function by adsorbing to the metal surface via the reactive headgroup, such that the hydrocarbon chains are left extending into the oil. The surfaces are then able to brush past each other with the sole consequence being the temporary bending of the alkyl chains, rather than surface wear ${ }^{1}$.

Saturated long-chain carboxylic acids such as palmitic acid $\left(\mathrm{CH}_{3}\left(\mathrm{CH}_{2}\right)_{14} \mathrm{CO}_{2} \mathrm{H}\right)$ have an illustrious history as effective friction modifiers. Their unsaturated counterparts, such as oleic (one double bond) and linoleic (two double bonds) acids, are also often found in lubricant mixtures, particularly in biobased feedstocks, most often in their naturally-occurring cis-form. It is, therefore, of interest to compare the adsorption behaviour of these molecules at a model engine/oil interface. In this work, this is represented using an iron oxide surface under a straight-chain alkane oil. It has been shown that the surface composition of a deposited film of iron on a silicon substrate, such as the ones used in this work, comprises a mixture of iron oxides, with the dominant phase thought to be $\alpha-\mathrm{Fe}_{2} \mathrm{O}_{3}$, also known as hematite ${ }^{2,3}$.

Three C18 fatty acids were used that differed only in the number of double bonds existing in their alkyl chains - stearic, oleic and linoleic acid - as shown in Figure 1. Their adsorption behaviour at the iron oxide/oil interface has been characterised using a combination of surface study techniques; depletion isotherms are used to compare the amounts adsorbed as a function of bulk composition and affinity for the surface ${ }^{4,5}$; polarised neutron reflectometry (PNR) is used to characterise the structure of adsorbed oleic acid molecules for comparison with previous studies of a saturated fatty 
acid $^{6-9}$, and sum frequency generation (SFG) spectroscopy proffers information concerning the molecular packing and orientation ${ }^{10}$.

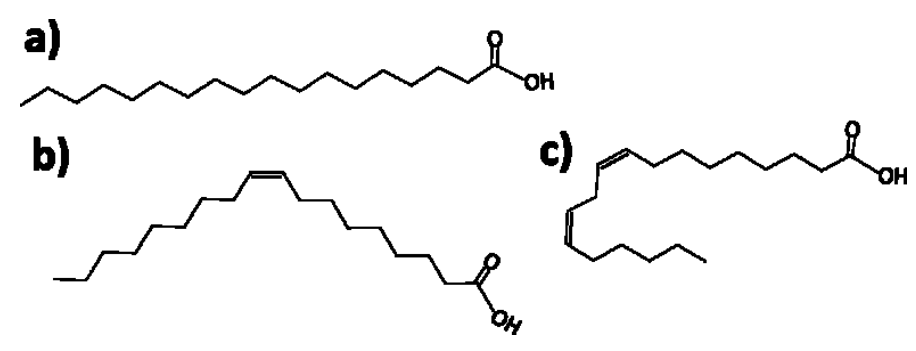

Figure 1. a) Stearic acid b) oleic acid c) linoleic acid

\section{Experimental}

\section{Materials}

Iron oxide powder was obtained from Sigma (> $99 \%$ purity, $69.7 \% \mathrm{Fe}$, by mass, determined by titration) and found to have a surface area of $4.15 \mathrm{~m}^{2} \mathrm{~g}^{-1}$ (determined by $\mathrm{N}_{2}$ adsorption fitted to the BET adsorption equation $\left.{ }^{11}\right)$. Dodecane was purchased from VWR (99\%) and all other chemicals from Sigma-Aldrich (all > $99 \%$ ). Iron/iron oxide films used for the PNR and SFG experiments were sputtered to a thickness of $100 \AA \AA$ onto polished silicon substrates, (111) orientation, (n)-type. For the PNR, the silicon substrate used was $55 \mathrm{~mm}$ in diameter and $5 \mathrm{~mm}$ thick, and deposition was carried out at the Nanoscience Centre, Cambridge by means of reactive magnetron sputtering ${ }^{12}$. For the SFG, substrates had dimensions $10 \times 10 \times 0.5 \mathrm{~mm}$, and deposition was effected by thermal evaporation at the Department of Chemistry, Cambridge.

\section{Depletion isotherms}

Depletion isotherms were measured by tumbling samples $(10 \mathrm{~mL})$ of the adsorbate in dodecane over a given concentration range $\left(0-2.5 \times 10^{-3} \mathrm{~mol} \mathrm{dm}^{-3}\right)$ with a high surface-area iron oxide powder $(0.5$ g). Samples were allowed to equilibrate over $24 \mathrm{~h}$, before centrifuging to separate the solid from the 
supernatant. The final acid concentration was evaluated by integration of carboxylic acid peak areas in the IR spectra and comparison to a calibrated set of standard concentrations.

PNR

The PNR experiments were conducted on the V6 instrument at the Helmholtz-Zentrum in Berlin. The wavelength was kept fixed at $4.66 \AA$, and incident beam angles varied by means of tilting the sample. Pyrolytic graphite crystals were used as a monochromator and polarisation achieved using supermirrors placed before the sample, which was kept in a magnetic field. Further instrumental details are published elsewhere ${ }^{13}$. A schematic of the cell set-up is shown in Figure 2; the solutions were pipetted in and out of a PTFE trough in contact with the sputtered substrate (cleaned before mounting by UV/ozone, 30 minutes). The iron/iron oxide layers were first characterised under the pure solvent, and then increasing concentrations of oleic acid added, with reflectivity profiles measured at each using both polarisations. The PNR data were fitted to a structural model using the Rascal fitting programme to minimise $\chi^{2}$.

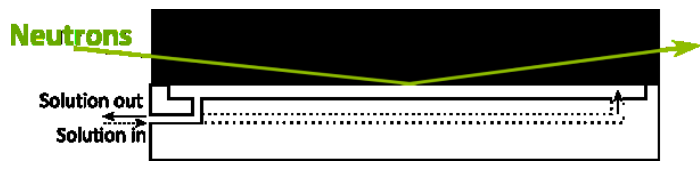

Figure 2. Side view of the cell set-up, with the film/silicon substrate held above the PTFE trough, secured by an outer aluminium casing and screws (not shown here for the purposes of clarity).

SFG

SFG spectra were recorded using an EKSPLA picosecond spectrometer (29 ps pulses at $50 \mathrm{~Hz}$ ) at the Department of Chemistry, Cambridge. The substrates were cleaned by UV/ozone (30 minutes) before mounting in a custom-made cell, with calcium fluoride prism. Spectra were recorded in PPP and SSP polarisation combinations (where ' $s$ ' and ' $p$ ' denote polarisation perpendicular and parallel to the plane of incidence respectively, and are given for the beams in the order: SFG, visible, IR) over 
the $\mathrm{C}-\mathrm{H}$ stretching region $\left(2800-3000 \mathrm{~cm}^{-1}\right)$. A co-propagating geometry was used, with angles of $53^{\circ}$ and $60^{\circ}$ to the surface normal for the IR and visible laser beams respectively. The spectra were normalised to the product of the IR and visible beam intensities using the EKSPLA normalisation facility, and fitted using the custom-built software, Baseline $2000^{14}$.

\section{Results and discussion}

\section{Depletion isotherms}

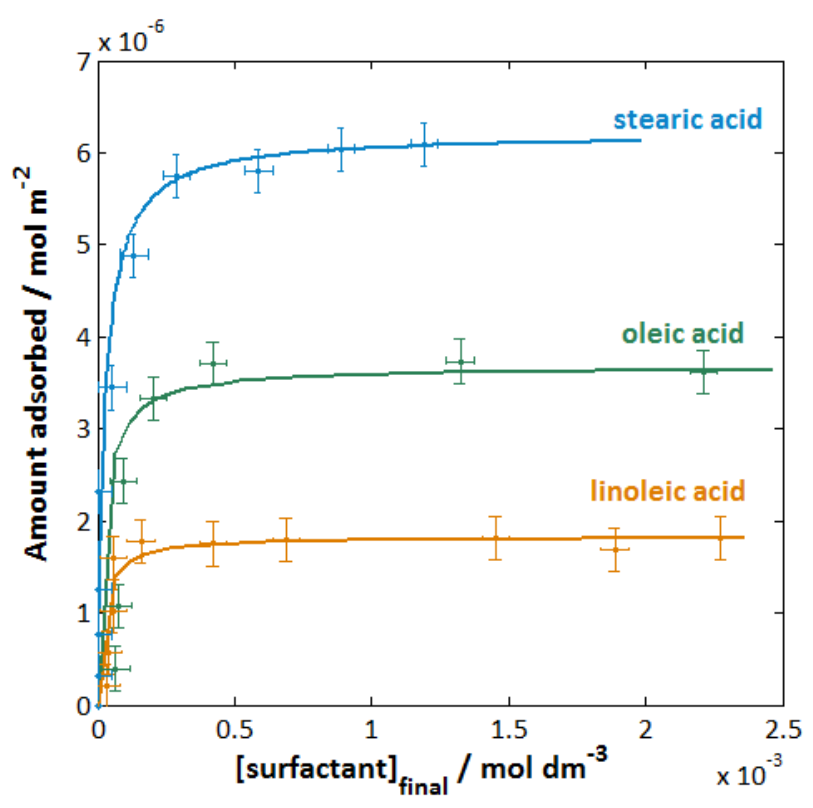

Figure 3. Depletion isotherms of stearic acid (blue), oleic acid (green) and linoleic acid (orange) on iron oxide powder from dodecane, with Langmuir fits shown as solid lines.

\begin{tabular}{|c|c|c|}
\hline & $v_{\max }$ & Plateau area \\
& $/ \mathrm{mol} \mathrm{m}^{-2}$ & per molecule \\
& & $/ \AA^{2}$ \\
\hline Stearic acid & $6.2( \pm 0.2) \times 10^{-6}$ & $27.5( \pm 2.0)$ \\
\hline Oleic acid & $3.7( \pm 0.2) \times 10^{-6}$ & $44.5( \pm 2.0)$ \\
\hline
\end{tabular}




\begin{tabular}{|l|l|l|}
\hline Linoleic acid & $1.8( \pm 0.2) \times 10^{-6}$ & $91.8( \pm 2.0)$ \\
\hline
\end{tabular}

Table 1. Langmuir fit parameters for the three acids.

Depletion isotherms for stearic, oleic and linoleic acids on iron oxide powder from dodecane are shown in Figure 3. Using a linear regression method, these were all found to give good agreement with the form of the Langmuir isotherm model (shown as solid lines in the figure), indicating monolayer adsorption, as would be expected. Fitted parameters for the three acids are shown in Table 1. There is a marked difference in the $v_{\max }$ values - the total amount adsorbed - which decreases as the number of double bonds increases. This is interpreted to arise from the lower packing density of unsaturated alkyl chains compared to the saturated analogues due to the additional disorder in the chain caused by the rigid double bonds. The $v_{\max }$ value for the saturated stearic acid, $6.2 \times 10^{-6} \mathrm{~mol} \mathrm{~m}^{-2}$, corresponds to an area per molecule of $27.5 \AA^{2}$ at the isotherm plateau, a little larger than the expected value ${ }^{15}$ for a fully-packed monolayer of around $20-22 \AA^{2}$. This can be partially explained by a limitation of available surface binding sites. A brief consideration of the assumed ideal surface structure of $\alpha-\mathrm{Fe}_{2} \mathrm{O}_{3}(0001)$ surface, in which the iron atoms are known ${ }^{16}$ to be separated by a distance of around $5 \AA$, verifies that an area per molecule of $27.5 \AA^{2}$ is reasonable for monolayer coverage, given that the surface area per binding site is around $25 \AA^{2}$, and allowing for some surface defects and packing disorder.

The plateau adsorption value for stearic acid is slightly less than that observed by Campana et al. for similar concentrations of palmitic acid on an iron oxide surface from hexadecane measured using neutron reflectometry ${ }^{17}$. They calculate an adsorbed amount of $2 \mathrm{mg} \mathrm{m}^{-2}$ when an initial concentration of $2 \mathrm{mM}$ is used, equivalent to around $7.8 \times 10^{-6} \mathrm{~mol} \mathrm{~m}^{-2}$. This variation could arise due to the assumption in the neutron reflectometry calculation of a completely flat surface; as the actual roughness for such substrates is generally in the region of 5-10 $\AA$, the real surface area is greater than that calculated, resulting in an overestimation of the plateau adsorption value ${ }^{3}$. Furthermore, there are likely to be significant differences between the surface sites available on the deposited film 
surface and powder surface, leading to varying adsorption behaviour. Nègre et al. report a plateau range of 5-6 $\times 10^{-6} \mathrm{~mol} \mathrm{~m}^{-2}$ for stearic acid on an iron oxide powder from a non-polar oil, and Studt observes a similar value for stearic acid on iron powder, both according well with the observations here $^{18,19}$.

Unusually, Wheeler et al. report a slightly higher adsorption plateau for oleic acid on an iron oxide powder from hexane compared to stearic acid, although a much lower plateau is seen for linoleic in agreement with the results reported here ${ }^{20}$. In addition, their areas per molecule are considerably higher $\left(>75 \AA^{2}\right.$ ) than those determined here, leading them to an unusual conclusion of flat molecular adsorption.

However, in general, surfactants with unsaturated chains are known to show a lesser affinity for the surface than their saturated counterparts, partially due to their less efficient packing density, as inferred here, but also to their frequently higher solubility in both aqueous and organic solvents, such that they are less likely to adsorb onto the surface ${ }^{21,22}$. Interestingly, Loehle et al. use computational modelling to predict that linoleic and oleic acids will preferentially adsorb on steel surfaces from a mixture including saturated fatty acids in a commercial oil. They attribute this to the higher diffusion coefficients of the unsaturated acids through the oil; as they are predicted to bind via irreversible chemisorption, it is clear that the first acid molecules to reach the surface - the unsaturated acids - will adsorb, such that the stearic acid arriving later will be unable to displace them $^{23}$. As saturated acids are known to be far more effective tribologically as friction modifiers ${ }^{24,25}$, this suggests that the best approach for a multi-component system would be to pretreat the surface with the saturated acid to prevent any detrimental effects on its beneficial lubricating behaviour by mixing in the adsorbed layer. It is presumed that the superior lubricating characteristics of the saturated acids arises purely from their ability to pack more efficiently at the surface, whereas the equilibrium surface concentrations of the unsaturated acids are necessarily lower. It would be of 
interest to compare the tribological behaviour of all three acids at a known equal surface concentration, which, based on these results, is likely to be similar.

PNR
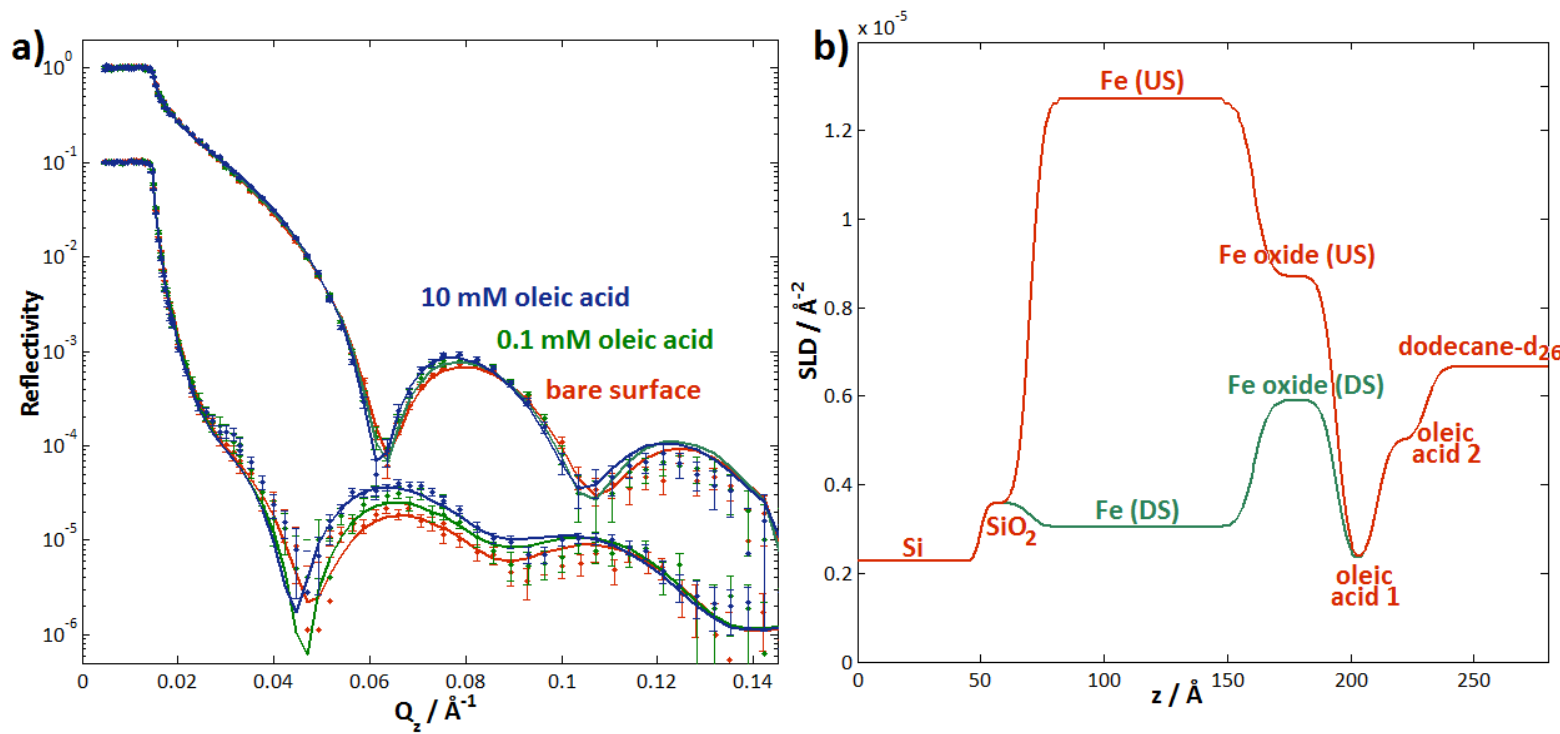

Figure 4. a) PNR profiles of the iron oxide/oleic acid/dodecane- $d_{26}$ system, with just the lowest and highest concentrations shown, as marked. (Data shown as points with fits as solid lines. The down-spin data are shown offset vertically by a factor of 10 for clarity.) b) Fitted SLD profiles for the $0.01 \mathrm{M}$ oleic acid system, showing the two acid layers (US = up-spin; DS = down-spin).

Adsorption of protonated oleic acid from dodecane- $\mathrm{d}_{26}$ onto deposited iron/iron oxide films on a silicon substrate was investigated using PNR. The iron film was first characterised under deuterated and protonated solvents, giving fitted thicknesses of 91 and $33 \AA$ for the iron and iron oxide layers respectively. Oleic acid adsorption was measured at concentrations of $0.1,1,4,7$ and $10 \times 10^{-3} \mathrm{~mol}$ $\mathrm{dm}^{-3}$. Representative fitted reflectivity profiles for three concentrations are shown in Figure 4a, with the fit parameters presented in Table 2. For $0.1-4 \times 10^{-3} \mathrm{~mol} \mathrm{dm}^{-3}$, the thickness of the acid was fitted to be $22 \AA( \pm 2 \AA)$, around the length of the extended molecule $(20 \AA)^{26}$, implying, as would be expected, that the molecule sits relatively upright on the surface. However, when increasing the concentration to $7 \times 10^{-3} \mathrm{~mol} \mathrm{dm}^{-3}$, the layer thickness was found to increase to 26 ( $\pm 2 \AA$ ) $\AA$, and for 
the $0.01 \mathrm{~mol} \mathrm{dm}$ concentration, it was necessary to include two layers to give a good fit, with the resultant SLD profile shown in Figure 4b.

The fitted layer thicknesses and solvation values were used to calculate the amounts adsorbed (shown in the final column of Table 2). These were calculated by relating the total volume, $V_{t}$, of a 1 $x 1 \mathrm{~m}$ box with thickness equal to that fitted for the layer to the number of oleic acid molecules, $\mathrm{N}_{\mathrm{A}}$, therein:

$$
V_{t}=N_{A} V_{A}+N_{S} V_{S}
$$

where $V_{A}$ and $V_{S}$ are the volumes of one oleic acid molecule and one solvent molecule respectively (calculated using given bulk densities of 0.895 and $0.864 \mathrm{~g} \mathrm{~mL}^{-1}$ for oleic acid and dodecane- $\mathrm{d}_{26}$ respectively) and $\mathrm{N}_{\mathrm{S}}$ is the number of solvent molecules in the box. The fractions of oleic acid and solvent for each concentration were taken from the fitted solvations, as presented in Table 2 .

\begin{tabular}{|c|c|c|c|c|}
\hline $\begin{array}{c}\text { [Oleic acid] } \\
/ \times 10^{-3} \mathbf{m o l ~ d m}^{-3}\end{array}$ & $\begin{array}{c}\text { Thickness } \\
/ \AA\end{array}$ & $\begin{array}{c}\text { Roughness } \\
/ \AA\end{array}$ & $\begin{array}{c}\text { Solvation } \\
/ \%\end{array}$ & $\begin{array}{c}\text { Amount adsorbed } \\
\text { calc. }\end{array}$ \\
\hline 0.1 & $22.0( \pm 2)$ & 6.0 & 45 & $4.39( \pm 0.45)$ \\
\hline 1 & $22.0( \pm 2)$ & 6.0 & 42 & $4.58( \pm 0.45)$ \\
\hline 4 & $22.0( \pm 2)$ & 6.0 & 38 & $4.84( \pm 0.45)$ \\
\hline 7 & $25.8( \pm 2)$ & 6.0 & 36 & $5.82( \pm 0.45)$ \\
\hline 10 & $17.2( \pm 2)$ & 2.0 & 25 & $6.60( \pm 0.45)$ \\
& $20.3( \pm 2)$ & 2.0 & 72 & \\
\hline
\end{tabular}

Table 2. Fitted PNR parameters for the oleic acid layers on the iron oxide surface, adsorbed from dodecane- $d_{26}$. At a concentration of $0.01 \mathrm{~mol} \mathrm{dm}^{-3}$, two layers were fitted, with individual parameters as shown; the amount adsorbed presented is the overall amount for both layers.

Part of the resultant data is shown in Figure 5 (points) with a Langmuir model (solid line). The $v_{\max }$ value of $4.8 \times 10^{-6} \mathrm{~mol} \mathrm{~m}^{-2}$ is somewhat higher than that predicted by the depletion isotherm results, 
also shown for comparison, a discrepancy also observed for the stearic acid results as recorded above, when compared to the neutron reflectometry data of Campana et al. This is partially attributed to the effects of roughness, given that the back-calculation from the PNR results assumes a perfectly flat surface, whereas in fact the iron oxide surface was modelled with a roughness of around $5 \AA$, as well as to differences between the deposited film, thought to contain a mixture of oxides $^{3}$, and the iron oxide powder, which is entirely $\mathrm{Fe}_{2} \mathrm{O}_{3}$; it is probable that the surface planes exposed in the two different materials will differ, leading to differences in surface energy and exposed binding sites. Importantly, the calculated adsorbed amount of oleic acid remains significantly lower than that seen for the saturated stearic acid, as predicted.

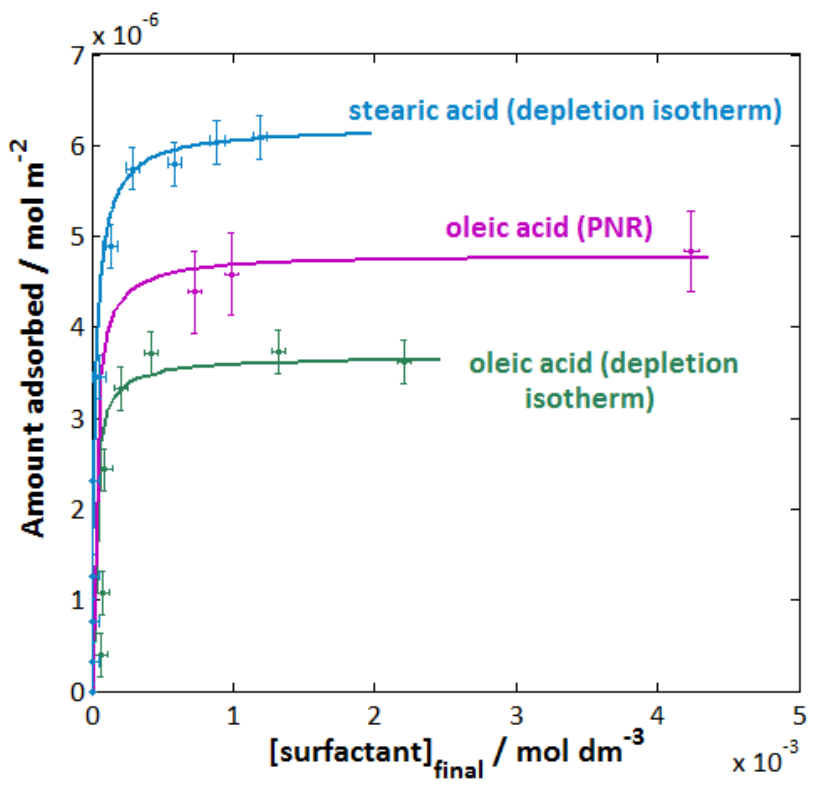

Figure 5. The isotherm of oleic acid on the deposited iron oxide film from the PNR results shown with the depletion isotherms of oleic acid and stearic acid for comparison. Data are shown as points with the Langmuir models as solid lines.

The two-layer model fitted for higher concentrations is in accord with the observations of Bronstein et al. who report two types of acid peak in the IR spectra for oleic acid adsorbed on iron oxide, with one disappearing upon washing ${ }^{27}$. This was further confirmed by an attempt to thoroughly 'wash' 
the deposited iron surface in this experiment by flushing the neutron reflectometry cell with copious solvent, after which a further reflectivity profile was measured. This is shown in Figure 6; it is clear that despite extensive rinsing, a layer of oleic acid still remains on the surface, although with less adsorbate remaining than seen at $0.01 \mathrm{~mol} \mathrm{dm}^{-3}$. In fact, the solvation value of the 'washed' surface was found to lie between that seen for 1 and $4 \times 10^{-3} \mathrm{~mol} \mathrm{dm}^{-3}$ oleic acid. Hence, it would appear that oleic acid adsorbs irreversibly at lower concentrations, probably via a chemisorbed Fe-O-C bond. At higher concentrations, a significant proportion of reversible adsorption, possibly physisorbed protonated acid, is seen that can be removed upon solvent washing.

Interestingly, Doig et al., who have performed molecular dynamics simulations on oleic and stearic acid/hematite/squalene systems, predict that the main difference arising from the unsaturated chain is to make the oleic acid sit more upright on the surface, whereas the stearic acid will sit at an angle ${ }^{28}$. This is supported by the PNR results reported here, which indicate a layer thickness close to that of the extended oleic acid molecule, whereas PNR layer thicknesses for palmitic acid suggest it sits at an angle ${ }^{17}$. 


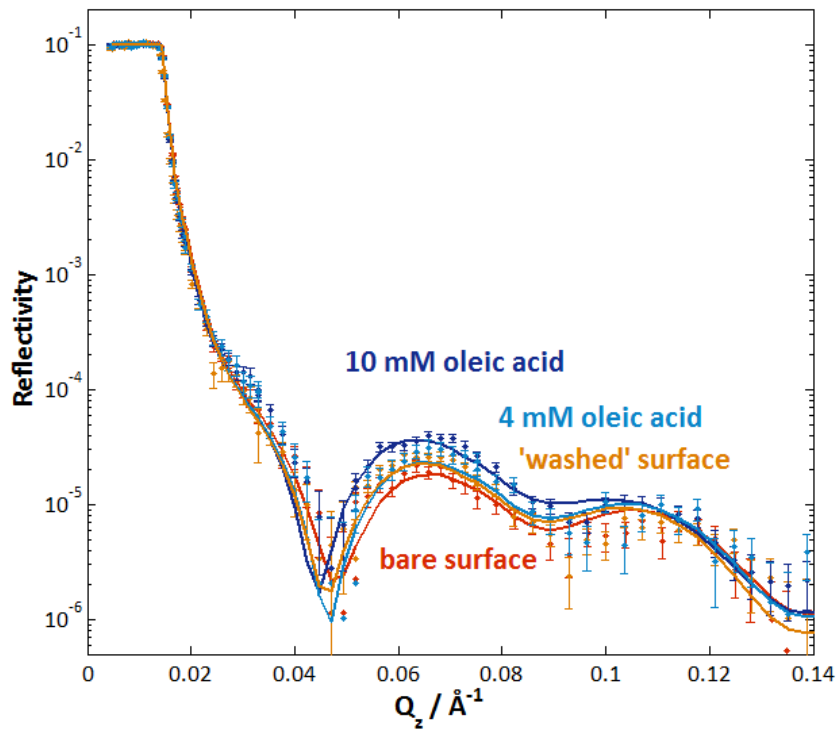

Figure 6. Comparison of PNR data (down-spin only) for $0.01 \mathrm{~mol} \mathrm{dm}^{-3}$ and $4 \times 10^{-3} \mathrm{~mol} \mathrm{dm}^{-3}$ oleic acid against the data for the bare surface and for the surface that has been washed copiously with solvent, as labelled, showing that the oleic acid cannot be fully removed, although some is lost upon washing. Data are shown as points with fits as solid lines.

SFG

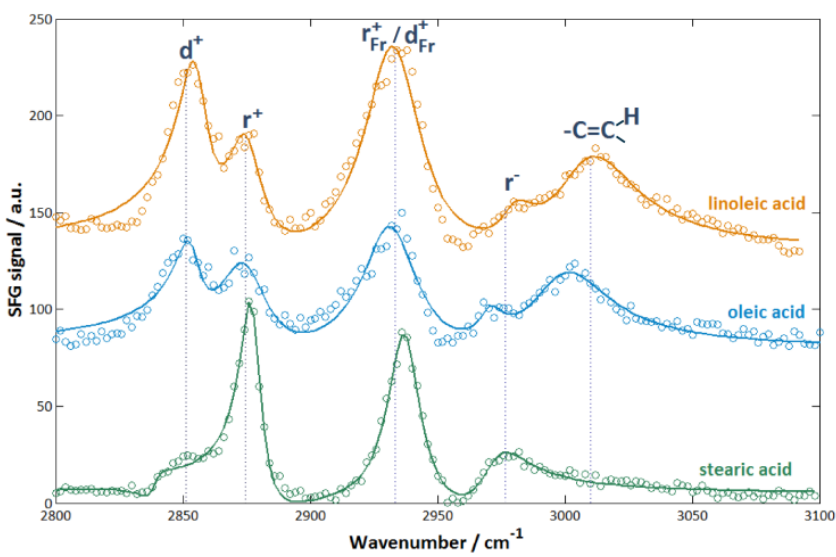

Figure 7. SSP spectra of stearic (green), oleic (blue) and linoleic (orange) acids on iron oxide from dodecane- $d_{26}$. Data offset vertically for clarity.

Figure 7 compares the SFG SSP spectra for the protonated stearic, oleic and linoleic acids from deuterated dodecane onto iron oxide films. Two significant observations can be made; firstly, the 
proportion of $\mathrm{d}^{+}$intensity (arising from the methylene groups along the alkyl chain backbone) increases with the extent of unsaturation in the acid, with a $\mathrm{d}^{+}: \mathrm{r}^{+} \%$ ratio of $0.15,96.9$ and $232 \%$ for stearic, oleic and linoleic acids respectively. This is unsurprising, since the locked double bonds in the unsaturated acids force a break in the centrosymmetric environment of the alkyl chain methylene groups. The higher degree of disorder in the chain packing, and hence increased effective surface space required, seen for the unsaturated acids doubtless explains their lower surface coverage in the depletion isotherms reported above.

The second observation of interest is the emergence of a peak around $3000 \mathrm{~cm}^{-1}$ for oleic and linoleic acid, which is attributed to the $\mathrm{C}-\mathrm{H}$ stretch from the double-bonded carbons ${ }^{29}$. However, as shown in Figure 8, for both oleic and linoleic acid this peak decreases in intensity proportionally to the other peaks upon heating the sample. For oleic, the fitted peak intensity diminishes from $7.5 \%$ of the total peak intensities at room temperature to $0.24 \%$ at $70^{\circ} \mathrm{C}$. Upon cooling the sample and remeasuring the spectrum, the intensity proportion remains at $0.24 \%$. A similar progressive decrease is seen for linoleic acid. The most probable explanation for this lies in the relative instability of unsaturated alkyl chains. A simple conclusion might be that the acids are undergoing hydrogenation across the double bond to form their saturated counterparts. However, this generally requires a catalyst such as nickel or ruthenium, and a plentiful supply of hydrogen ${ }^{30}$, neither of which are present in this system. Similarly, other common reactions such as oxidative cleavage or esterification require particular reagents absent here. For example, Voss et al. use SFG to study oxidation by ozone-treatment of oleic acid adsorbed at the air/water interface, and see a gradual decrease in the $r^{+}$peak intensity with respect to the $d^{+31}$. In contrast, the results reported here suggest rather an increase in ordering, as the $\mathrm{d}^{+}: \mathrm{r}^{+}$ratio for oleic acid decreases from $96.9 \%$ at room temperature to $33.6 \%$ for the final cooled films, although any ratio change is essentially negligible for linoleic acid. 
A more probable explanation, therefore, is the possibility of heat-induced isomerisation. Hadaruga et al. report heat-treatment of oleic acid over a range of temperatures; an analysis of the degradation products found that by $150^{\circ} \mathrm{C}$ the proportion of oleic acid had dropped to $82 \%$, with 8 $\%$ of the trans-isomer, elaidic acid, present and $7 \%$ 10-undecanoic acid ester derivative ${ }^{32}$. Furthermore, Li et al. report the detection of marginal amounts of the trans-isomers of linoleic acid in soybean oils samples upon heating to 180 and $220^{\circ} \mathrm{C}^{33}$.

If a fraction of the adsorbed oleic or linoleic acid molecules are indeed undergoing isomerisation upon heating to form their trans counterparts, and potentially other products, the proportional decrease of the double-bond intensity and increase in ordering (for the oleic acid sample) could be explained by a reorientation of some of the double bond groups such that their signal cancels out those arising from the original cis-isomers. Trans-isomers would also be able to pack on the surface with fewer gauche defects; the unchanged $\mathrm{d}^{+}: \mathrm{r}^{+}$ratio for the linoleic acid may arise because only one double bond is isomerised, with little consequent effect on chain ordering.
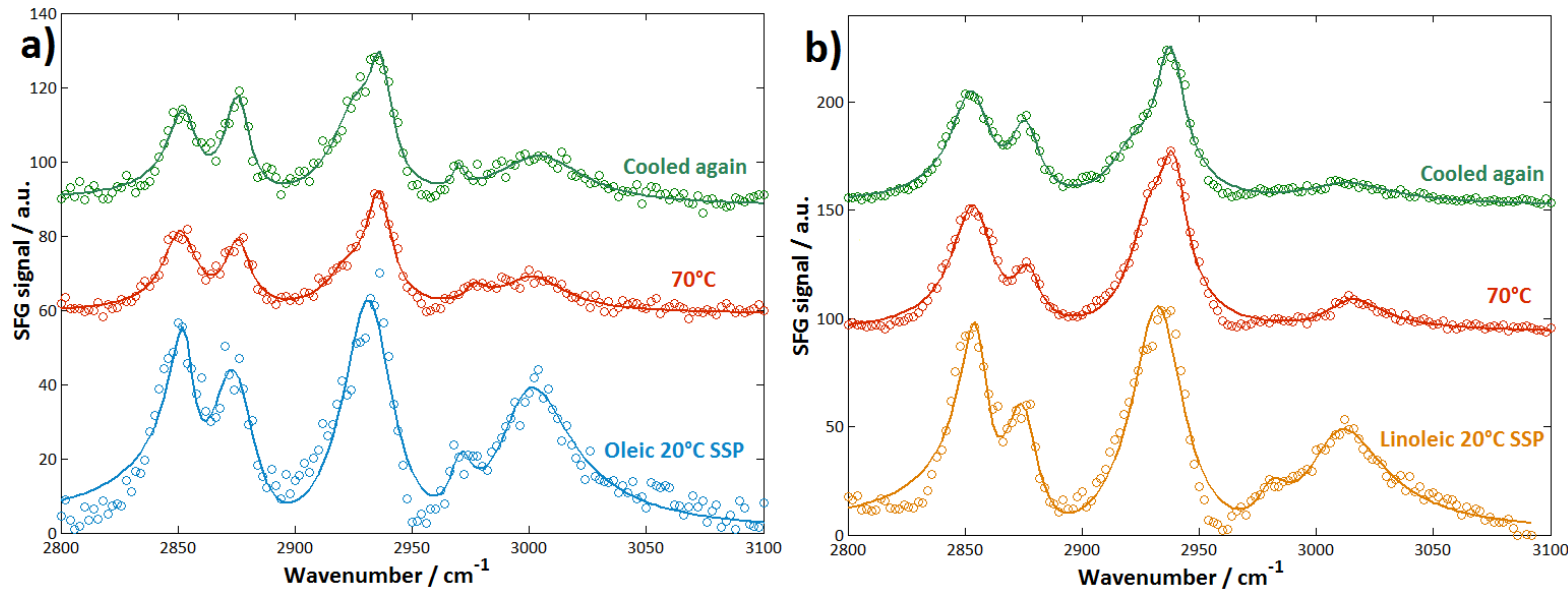

Figure 8. SSP SFG spectra for a) oleic and b) linoleic acid at increasing temperatures and once cooled again after heating, as labelled. In both cases, a significant diminishing of the peak pertaining to the double bond $\mathrm{C}-\mathrm{H}$ stretch is seen.

\section{Conclusions}


The depletion isotherms showed a clear trend of increasing adsorption for the acids with less unsaturation in their alkyl chains, implying that coverage was reliant on the ability of the carbon chains to pack well. This was supported by the SFG data, which demonstrated an increase in gauche defects for the unsaturated fatty acids, indicated by the higher proportional intensities of the $\mathrm{d}^{+}$ (methylene) peaks. The PNR results for oleic acid yielded an isotherm in accordance with this trend, despite the errors of over-estimation of adsorbed amount incurred by assuming a completely flat surface and differences between the deposited films and powder surfaces. A $22 \AA$ layer was fitted, implying non-tilted adsorption, in contrast to that seen for palmitic acid, with a further diffuse layer seen at higher $(0.01 \mathrm{M})$ concentrations. Upon washing, the additional layer was removed, but the initial layer remained, suggesting it was bound irreversibly.

SFG spectra of the adsorbed oleic and linoleic acids taken at increased temperatures $\left(70^{\circ} \mathrm{C}\right)$ showed a decrease in intensity for the peak around $3000 \mathrm{~cm}^{-1}$ pertaining to the double bond $\mathrm{C}-\mathrm{H}$ stretch, with this effect remaining upon recooling. This was attributed to partial isomerisation of the double bonds to form trans-isomers.

These results may therefore shed some light on the observations that unsaturated fatty acid additives provide a less effective surface protection than their saturated counterparts; slimmer molecules - i.e. those with no branching or cyclic components - are known to afford better lubrication, thought to be due to more efficient surface packing ${ }^{19,34}$. The SFG results here show that unsaturated fatty acids do not pack as well at the surface, and the depletion isotherms confirm that their overall maximum adsorbed amount is lower than that of the saturated acid. It has been reported that monolayer coverage is necessary for good friction reduction; intermediate coverage of palmitic acid on an iron oxide surface may actually cause an increase in wear as wettability of the surface by the bulk oil is decreased ${ }^{35}$. Hence, the unsaturated fatty oils found in biobased lubricants such as vegetable oils may even have a detrimental effect on the surface, particularly if they are able 
to reach the surface first and bind irreversibly, as seems likely. On the reverse side, these results may also aid in understanding the superior ability of oleic acid to stabilise iron oxide nanoparticles, since this application has the opposite requirement, favouring rather bulky groups with low packing density per carboxylic acid headgroup ${ }^{36-38}$.

\section{Acknowledgements}

Mary Wood is grateful for funding from the Oppenheimer Trust. The PNR data were collected using the V6 instrument at the Helmholtz-Zentrum Berlin (experiment number MAT-04-2131).

\section{Bibliography}

(1) Crawford, J.; Psaila, A.; Orszulik, S. T. In Chemistry and technology of lubricants; Mortier, R. M., Orszulik, S. T., Eds.; Blackie Academic and Professional: Great Britain, 1992.

(2) Aronniemi, M.; Lahtinen, J.; Hautojärvi, P. Characterisation of iron oxide films. Surf. Int. Anal. 2004, 36, 1004-1006.

(3) Wood, M. H.; Welbourn, R. J. L.; Charlton, T.; Zarbakhsh, A.; Casford, M. T.; Clarke, S. M. Hexadecylamine adsorption at the iron oxide-oil interface. Langmuir 2013, 29, 13735-13742.

(4) Okeola, F. O.; Odebunmi, E. O. Freundich and Langmuir isotherm parameters for adsorption of methylene blue by activated carbon derived from agrowastes. Ads. J. Int. Ads. Soc. 2010, 4, 281-288.

(5) Giles, C. H.; Smith, D.; Huitson, A. A general treatment and classification of the solute adsorption isotherm. J. Coll. Int. Sci. 1974, 47, 755-765.

(6) Penfold, J.; Thomas, R. K. The application of the specular reflection of neutrons to the study of surfaces and interfaces. J. Phys. Condens. Matter 1990, 2, 1369-1412.

(7) Ankner, J. F.; Felcher, G. P. Polarised neutron reflectometry. J. Magn. Magn. Mater. 1999, 200, 741-754.

(8) Penfold, J.; Thomas, R. K.; Lu, J. R.; Staples, E.; Tucker, I.; Thompson, L. The study fo surfactant adsorption by specular neutron reflection. Physica B. 1994, 198, 110-115. 
(9) Penfold, J.; Thomas, R. K. In Advanced Chemistry of Monolayers at Interfaces; Elsevier Ltd.: London, 2007.

(10) Lambert, A.; Davies, P.; Neivandt, D. Implementing the theory of sum frequency generation vibrational spectroscopy: a tutorial review. Appl. Spec. Rev. 2005, 40, 103-145.

(11) Brunauer, S.; Emmett, P. H.; Teller, E. Adsorption of gases in multimolecular layers. J. Am. Chem. Soc. 1938, 60, 309-319.

(12) Penning, F. M.; Moubis, J. H. A. Cathode sputtering in a magnetic field. Proc. Kon. Ned. Akad. Wetensch. 1940, 43, 41-56.

(13) Paul, A.; Krist, T.; Teichert, A.; Steitz, R. Specular and off-specular scattering with polarisation and polarisation analysis on reflectometer V6 at BER II, HZB. Physica B 2011, 406, 1598-1606.

(14) Lambert, A. G. Resonantly enhanced sum frequency spectroscopy of adsorption on hydrophilic mica substrates, PhD Thesis, University of Cambridge, 2001.

(15) Mann, S.; Heywood, B. R.; Rajam, S.; Birchall, J. D. Controlled crystallisation of $\mathrm{CaCO}_{3}$ under stearic acid monolayers. Nature 1988, 334, 692-695.

(16) Shaikhutdinov, S. K.; Joseph, Y.; Kuhrs, C.; Ranke, W.; Weiss, W. Structure and reactivity of iron oxide structures. Faraday. Discuss. 1999, 114, 363-380.

(17) Campana, M.; Teichert, A.; Clarke, S.; Steitz, R.; Webster, J. R. P.; Zarbakhsh, A. Surfactant adsorption at the metal-oil interface Langmuir 2011, 27, 6085-6090.

(18) Nègre, J. C.; Denoyel, R.; Rouquerol, F.; Rouquerol, J. In Interactions solide-liquide dans les milieux poreux; Editions Technip: Paris, 1985.

(19) Studt, P. Boundary lubrication: adsorption of oil additives on steel and ceramic surfaces and its influence on friction and wear. Tribol. Int. 1989, 22, 111-119.

(20) Wheeler, D. H.; Potente, D.; Wittcoff, H. Adsorption of dimer, trimer, stearic, oleic, linoleic, nonanoic and azelaic acids on ferric oxide. J. Am. Oil Chem. Soc. 1971, 48, 125-128.

(21) Encyclopedia of Surface and Colloid Science; Somasundaran, P., Ed.; CRC Press, 2006.

(22) Froth Flotation; Fuerstenau, M. C., Jameson, G., Yoon, R.-H., Eds.; Society for Mining, Metallurgy and Exploration, Inc. (SME): Colorado, USA, 2007.

(23) Loehle, S.; Matta, C.; Minfray, C.; Le Mogne, T.; Martin, J.-M.; lovine, R.; Obara, Y.; Miura, R.; Miyamoto, A. Mixed lubrication with C18 fatty acids: effect of unsaturation. Tribol. Lett. 2014, $53,319-328$.

(24) Campen, S.; Green, J.; Lamb, G.; Atkinson, D.; Spikes, H. On the increase in boundary friction with sliding speed. Tribol. Lett. 2012, 48, 237-248.

(25) Kondo, H. Effect of double bonds on friction in the boundary lubrication of magnetic thin film media. Wear 1997, 202, 149-153. 
(26) Iwahashi, M.; Kasahara, Y.; Matsuzawa, H.; Yagi, K.; Nomura, K.; Terauchi, H.; Ozaki, Y.; Suzuki, M. Self-diffusion, dynamical molecular conformation, and liquid structures of $n$ saturated and unsaturated fatty acids. J. Phys. Chem. B 2000, 104, 6186-6194.

(27) Bronstein, L. M.; Huang, X.; Retrum, J.; Schmucker, A.; Pink, M.; Stein, B. D.; Dragnea, B. Influence of iron oleate complex structure on iron oxide nanoparticle formation. Chem. Mater. 2007, 19, 3624-3632.

(28) Doig, M.; Warrens, C. P.; Camp, P. J. Structure and friction of stearic acid and oleic acid films adsorbed on iron oxide surfaces in squalene. Langmuir 2014, 30, 186-195.

(29) Kleber, J.; Laß, K.; Friedrichs, G. Quantitative time-resolved vibrational sum frequency generation spectroscopy as a tool for thin film kinetic studies: new insights into oleic acid monolayer oxidation. J. Phys. Chem. A 2013, 117, 7863-7875.

(30) Mendes, M. J.; Santos, O. A. A.; Jordão, E.; Silva, A. M. Hydrogenation of oleic acid over ruthenium catalysts. Appl. Catal. A 2001, 217, 253-262.

(31) Voss, L. F.; Bazerbashi, M. F.; Beekman, C. P.; Hadad, C. M.; Allen, H. C. Oxidation of oleic acid at air/liquid interfaces. J. Geophys. Res. 2007, 112, D06209 1-9.

(32) Hadaruga, D. I.; Hadaruga, N. G.; Hermenean, A.; Rivis, A.; Paslaru, V.; Codina, G. Bionanomaterials: thermal stability of the oleic acid/ $\alpha$ - and $\beta$-cyclodextrin complexes. Rev. Chim. 2008, 59, 994-998.

(33) Li, A.; Yuan, B.; Li, W.; Wang, F.; Ha, Y. Thermally induced isomerisation of linoleic acid in soybean oiil. Chem. Phys. Lipids 2013, 166, 55-60.

(34) Papay, A. G. Oil soluble friction reducers - theory and application. J. Am. Soc. Lubr. Eng. 1983, 39, 419-426.

(35) Kajdas, C.; Majzner, M. Boundary lubrication of low-sulfur diesel fuel in the presence of fatty acids. Lubr. Sci. 2001, 14, 83-108.

(36) Gupta, A. K.; Gupta, M. Synthesis and surface engineering of iron oxide nanoparticles for biomedical applications. Biomat. 2005, 26, 3995-4021.

(37) Wu, W.; He, Q.; Jiang, C. Magnetic iron oxide nanoparticles: synthesis and surface functionalization strategies. Nanoscale Res. Lett. 2008, 3, 397-415.

(38) Hofmann, A.; Thierbach, S.; Semisch, A.; Hartwig, A.; Taupitz, M.; Rühl, E.; Graf, C. Highly monodisperse water-dispersable iron oxide nanoparticles for biomedical applications $J$. Mater. Chem. 2010, 20, 7842-7853. 

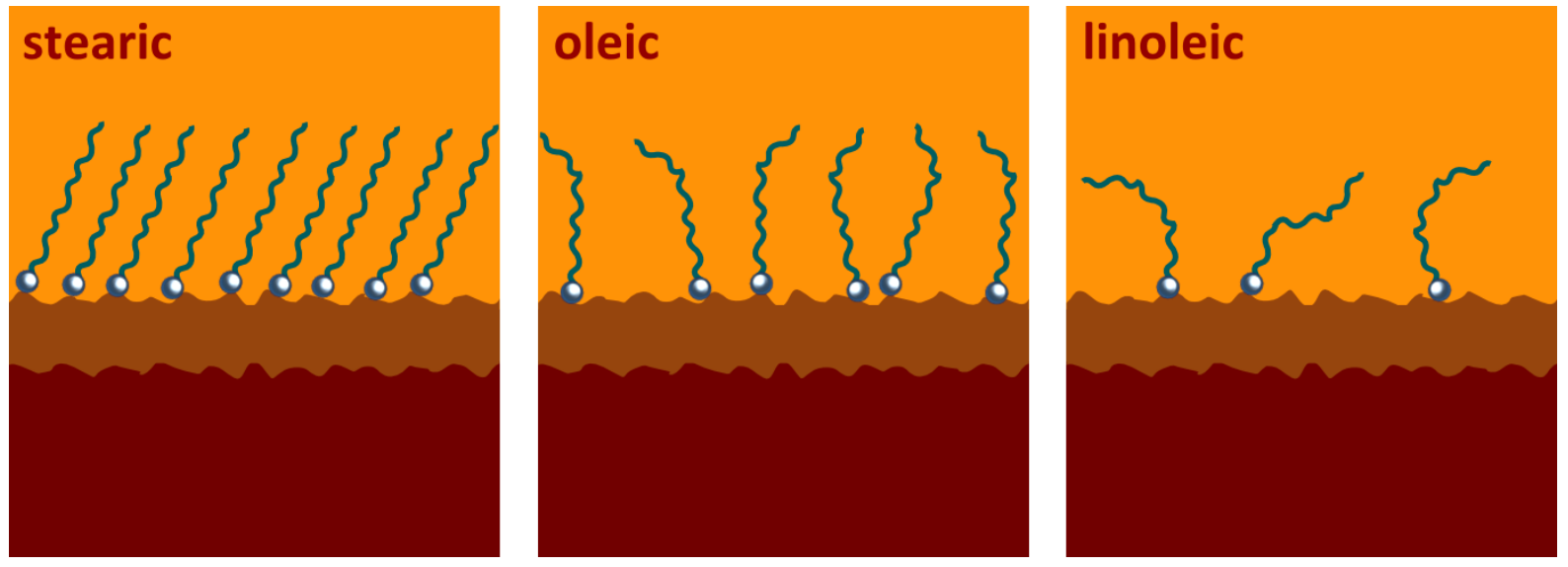